\title{
Principal Functions of Nonselfadjoint Discrete Dirac Equations with Spectral Parameter in Boundary Conditions
}

\author{
Yelda Aygar, ${ }^{1}$ Murat Olgun, ${ }^{1}$ and Turhan Koprubasi ${ }^{2}$ \\ ${ }^{1}$ Department of Mathematics, Ankara University, Tandogan, 06100 Ankara, Turkey \\ ${ }^{2}$ Department of Mathematics, Kastamonu University, 37100 Kastamonu, Turkey \\ Correspondence should be addressed to Yelda Aygar, yaygar@science.ankara.edu.tr \\ Received 18 July 2012; Accepted 12 September 2012 \\ Academic Editor: Patricia J. Y. Wong
}

Copyright (c) 2012 Yelda Aygar et al. This is an open access article distributed under the Creative Commons Attribution License, which permits unrestricted use, distribution, and reproduction in any medium, provided the original work is properly cited.

Let $L$ denote the operator generated in $\ell_{2}\left(\mathbb{N}, \mathbb{C}^{2}\right)$ by $a_{n+1} y_{n+1}^{(2)}+b_{n} y_{n}^{(2)}+p_{n} y_{n}^{(1)}=\lambda y_{n}^{(1)}, a_{n-1} y_{n-1}^{(1)}+$ $b_{n} y_{n}^{(1)}+q_{n} y_{n}^{(2)}=\lambda y_{n}^{(2)}, n \in \mathbb{N}$, and the boundary condition $\left(\gamma_{0}+\gamma_{1} \lambda\right) y_{1}^{(2)}+\left(\beta_{0}+\beta_{1} \lambda\right) y_{0}^{(1)}=\mathbf{0}$, where $\left(a_{n}\right),\left(b_{n}\right),\left(p_{n}\right)$, and $\left(q_{n}\right), n \in \mathbb{N}$ are complex sequences, $\gamma_{i}, \beta_{i} \in \mathbb{C}, i=0,1$, and $\lambda$ is an eigenparameter. In this paper we investigated the principal functions corresponding to the eigenvalues and the spectral singularities of $L$.

\section{Introduction}

Consider the boundary value problem (BVP)

$$
\begin{gathered}
-y^{\prime \prime}+q(x) y=\lambda^{2} y, \quad 0 \leq x<\infty, \\
y(0)=0,
\end{gathered}
$$

in $L^{2}\left(\mathbb{R}_{+}\right)$, where $q$ is a complex-valued function and $\lambda \in \mathbb{C}$ is a spectral parameter. The spectral theory of the above BVP with continuous and point spectrum was investigated by NaImark [1]. He showed the existence of the spectral singularities in the continuous spectrum of (1.1). Note that the eigen and associated functions corresponding to the spectral singularities are not the elements of $L^{2}\left(\mathbb{R}_{+}\right)$.

In $[2,3]$ the effect of the spectral singularities in the spectral expansion in terms of the principal vectors was considered. Some problems related to the spectral analysis of 
difference equations with spectral singularities were discussed in [4-7]. The spectral analysis of eigenparameter dependent nonselfadjoint difference equation was studied in $[8,9]$.

Let us consider the nonselfadjoint BVP for the discrete Dirac equations

$$
\begin{gathered}
a_{n+1} y_{n+1}^{(2)}+b_{n} y_{n}^{(2)}+p_{n} y_{n}^{(1)}=\lambda y_{n}^{(1)}, \quad n \in \mathbb{N}, \\
a_{n-1} y_{n-1}^{(1)}+b_{n} y_{n}^{(1)}+q_{n} y_{n}^{(2)}=\lambda y_{n}^{(2)}, \quad n \in \mathbb{N}, \\
\left(\gamma_{0}+\gamma_{1} \lambda\right) y_{1}^{(2)}+\left(\beta_{0}+\beta_{1} \lambda\right) y_{0}^{(1)}=0, \quad \gamma_{0} \beta_{1}-\gamma_{1} \beta_{0} \neq 0, \quad \gamma_{1} \neq a_{0}^{-1} \beta_{0},
\end{gathered}
$$

where $\left(\begin{array}{l}y_{n}^{(1)} \\ y_{n}^{(2)}\end{array}\right), n \in \mathbb{N}$ are vector sequences, $a_{n} \neq 0, b_{n} \neq 0$ for all $n \in \mathbb{N}, \gamma_{i}, \beta_{i} \in \mathbb{C}$, and $i=0,1$ and, $\lambda$ is a spectral parameter.

In [10] the authors proved that eigenvalues and spectral singularities of (1.2)-(1.3) have a finite number with finite multiplicities, if the condition,

$$
\sum_{n=1}^{\infty} \exp \left(\varepsilon n^{\delta}\right)\left(\left|1-a_{n}\right|+\left|1+b_{n}\right|+\left|p_{n}\right|+\left|q_{n}\right|\right)<\infty
$$

holds, for some $\varepsilon>0$ and $1 / 2 \leq \delta<1$.

In this paper, we aim to investigate the principal functions corresponding to the eigenvalues and the spectral singularities of the BVP (1.2)-(1.3).

\section{Discrete Spectrum of (1.2)-(1.3)}

Let for some $\varepsilon>0$ and $1 / 2 \leq \delta<1$,

$$
\sum_{n=1}^{\infty} \exp \left(\varepsilon n^{\delta}\right)\left(\left|1-a_{n}\right|+\left|1+b_{n}\right|+\left|p_{n}\right|+\left|q_{n}\right|\right)<\infty
$$

be satisfied. It has been shown that [10] under the condition (2.1), (1.2) has the solution

$$
\begin{gathered}
f_{n}(z)=\left(\begin{array}{c}
f_{n}^{(1)}(z) \\
f_{n}^{(2)}(z)
\end{array}\right)=\alpha_{n}\left(I+\sum_{m=1}^{\infty} A_{n m} e^{i m z}\right)\left(\begin{array}{c}
e^{i z / 2} \\
-i
\end{array}\right) e^{i n z}, \quad n=1,2, \ldots, \\
f_{0}^{(1)}(z)=\alpha_{0}^{11}\left\{e^{i z / 2}\left[1+\sum_{m=1}^{\infty} A_{0 m}^{11} e^{i m z}\right]-i \sum_{m=1}^{\infty} A_{0 m}^{12} e^{i m z}\right\},
\end{gathered}
$$

for $\lambda=2 \sin (z / 2)$ and $z \in \overline{\mathbb{C}}_{+}:=\{z \in \mathbb{C}: \operatorname{Im} z \geq 0\}$, where

$$
\alpha_{n}=\left(\begin{array}{cc}
\alpha_{n}^{11} & \alpha_{n}^{12} \\
\alpha_{n}^{21} & \alpha_{n}^{22}
\end{array}\right), \quad I=\left(\begin{array}{ll}
1 & 0 \\
0 & 1
\end{array}\right), \quad A_{n m}=\left(\begin{array}{cc}
A_{n m}^{11} & A_{n m}^{12} \\
A_{n m}^{21} & A_{n m}^{22}
\end{array}\right) .
$$


Note that $\alpha_{n}^{i j}$ and $A_{n m}^{i j}(i, j=1,2)$ are uniquely expressed in terms of $\left(a_{n}\right),\left(b_{n}\right),\left(p_{n}\right)$, and $\left(q_{n}\right), n \in \mathbb{N}$ as follows

$$
\begin{aligned}
& \alpha_{n}^{11}=\left[\prod_{k=n+1}^{\infty}(-1)^{n-k} b_{k} a_{k-1}\right]^{-1} \\
& \alpha_{n}^{12}=0, \\
& \alpha_{n}^{22}=\left[b_{n} \prod_{k=n+1}^{\infty}(-1)^{n-k+1} b_{k} a_{k-1}\right]^{-1}, \\
& \alpha_{n}^{21}=\alpha_{n}^{22}\left[p_{n}+\sum_{k=n+1}^{\infty}\left(p_{k}+q_{k}\right)\right] \\
& A_{n 1}^{12}=-\sum_{k=n+1}^{\infty}\left(p_{k}+q_{k}\right) \text {, } \\
& A_{n 1}^{11}=\sum_{k=n+1}^{\infty}\left[a_{k+1} a_{k}+b_{k}^{2}-p_{k} q_{k}+\left(p_{k}+q_{k}\right) A_{k 1}^{12}-2\right], \\
& A_{n 1}^{22}=-1+a_{n+1} a_{n}+\left(A_{n 1}^{12}\right)^{2}+A_{n 1}^{11} \\
& A_{n 1}^{21}=-\sum_{k=n}^{\infty}\left\{( q _ { k + 1 } + A _ { k 1 } ^ { 1 2 } ) \left[a_{k+1} a_{k}+q_{k+1}\left(p_{k+1}+q_{k+1}\right)+q_{k+1} A_{k 1}^{12}\right.\right. \\
& \left.\left.+b_{k+1}^{2}+A_{k+1,1}^{11}-1\right]-A_{k 1}^{12}\left(1+A_{k 1}^{11}\right)\right\}+\sum_{k=n+1}^{\infty}\left(q_{k} A_{k 1}^{22}-b_{k}^{2} p_{k}\right), \\
& A_{n 2}^{12}=-a_{n+1} a_{n}\left(q_{n+1}+A_{n 1}^{12}\right)+A_{n 1}^{12} A_{n 1}^{11}+A_{n 1}^{12}-A_{n 1}^{21} \\
& A_{n 2}^{11}=\sum_{k=n+1}^{\infty}\left\{\left(b_{k}^{2}-1\right) A_{k 1}^{11}-a_{k+1} a_{k}\left[\left(q_{k+1}+A_{k 1}^{12}\right) A_{k+1,1}^{12}-A_{k+1,1}^{22}\right]\right. \\
& \left.-\left(p_{k}-A_{k 1}^{12}\right)\left[q_{k} A_{k 1}^{11}+A_{k 1}^{12}-A_{k 2}^{12}\right]-q_{k} A_{k 1}^{21}+A_{k 1}^{12} A_{k 2}^{12}-A_{k 1}^{22}\right\}, \\
& A_{n 2}^{22}=-a_{n+1} a_{n}\left(q_{n+1}+A_{n 1}^{12}\right) A_{n+1,1}^{12}+a_{n+1} a_{n} A_{n+1,1}^{22}+A_{n 1}^{12} A_{n 2}^{12}-A_{n 1}^{11}+A_{n 2}^{11} \\
& A_{n 2}^{21}=\sum_{k=n}^{\infty}\left\{A_{k 1}^{12} A_{k 2}^{11}+\mathrm{A}_{k 2}^{21}-a_{k+1} a_{k}\left[\left(q_{k+1}+A_{k 1}^{12}\right) A_{k+1,1}^{11}-A_{k+1,1}^{21}\right]\right\} \\
& -\sum_{k=n+1}^{\infty}\left[\left(q_{k}+A_{k-1,1}^{12}\right)\left(q_{k} A_{k 2}^{12}-A_{k 1}^{11}+A_{k 2}^{11}\right)+b_{k}^{2} A_{k 2}^{21}-p_{k} A_{k 2}^{22}+A_{k 1}^{21}\right]
\end{aligned}
$$


and for $m \geq 3$

$$
\begin{aligned}
A_{n m}^{12}= & -a_{n+1} a_{n}\left[\left(q_{n+1}+A_{n 1}^{12}\right) A_{n+1, m-2}^{11}+A_{n+1, m-2}^{21}\right] \\
& +A_{n 1}^{12} A_{n, m-1}^{11}+A_{n, m-1}^{12}-A_{n, m-1}^{21}, \\
A_{n m}^{11}= & -\sum_{k=n+1}^{\infty} a_{k+1} a_{k}\left[\left(q_{k+1}+A_{k 1}^{12}\right) A_{k+1, m-1}^{12}-A_{k+1, m-1}^{22}\right] \\
& -\sum_{k=n+1}^{\infty}\left(p_{k}-A_{k 1}^{12}\right)\left(q_{k} A_{k, m-1}^{11}+A_{k, m-1}^{12}-A_{k m}^{12}\right)+\sum_{k=n+1}^{\infty}\left(b_{k}^{2}-1\right) A_{k, m-1}^{11} \\
& -\sum_{k=n+1}^{\infty} q_{k} A_{k, m-1}^{21}+\sum_{k=n+1}^{\infty} A_{k 1}^{12} A_{k m}^{12}-\sum_{k=n+1}^{\infty} A_{k, m-1}^{22}, \\
A_{n m}^{22}= & -a_{n+1} a_{n}\left[\left(q_{n+1}+A_{n 1}^{12}\right) A_{n+1, m-1}^{11}-A_{n+1, m-1}^{22}\right]+A_{n 1}^{12} A_{n m}^{12}+A_{n m}^{11}-A_{n, m-1}^{11}, \\
A_{n m}^{21}= & -\sum_{k=n}^{\infty} a_{k+1} a_{k}\left[\left(q_{k+1}+A_{k 1}^{12}\right) A_{k+1, m-1}^{11}-A_{k+1, m-1}^{21}\right] \\
& -\sum_{k=n+1}^{\infty}\left(q_{k}-A_{k-1,1}^{12}\right)\left(q_{k} A_{k m}^{21}+A_{k, m-1}^{11}-A_{k m}^{22}\right)-\sum_{k=n+1}^{\infty}\left(b_{k}^{2}-1\right) A_{k m}^{12} \\
& +\sum_{k=n}^{\infty} A_{k 1}^{12} A_{k m}^{22}+\sum_{k=n+1}^{\infty} q_{k} A_{k m}^{22}+\sum_{k=n}^{\infty} A_{k m}^{12}-\sum_{k=n+1}^{\infty} A_{k, m-1}^{21} .
\end{aligned}
$$

Moreover

$$
\left|A_{n m}^{i j}\right| \leq C \sum_{k=n+[|m / 2|]}^{\infty}\left(\left|1-a_{k}\right|+\left|1+b_{k}\right|+\left|p_{k}\right|+\left|q_{k}\right|\right)
$$

holds, where $[|m / 2|]$ is the integer part of $m / 2$ and $C>0$ is a constant. Therefore $f_{n}$ is vectorvalued analytic function with respect to $z$ in $\mathbb{C}_{+}:=\{z \in \mathbb{C}: \operatorname{Im} z>0\}$ and continuous in $\overline{\mathbb{C}}_{+}$ [10]. The solution $f(z)=\left(f_{n}(z)\right)=\left(\begin{array}{l}f_{n}^{(1)}(z) \\ f_{n}^{(2)}(z)\end{array}\right)$ is called Jost solution of (1.2).

Let us define

$$
F(z)=\left(\gamma_{0}+2 \gamma_{1} \sin \frac{z}{2}\right) f_{1}^{(2)}(z)+\left(\beta_{0}+2 \beta_{1} \sin \frac{z}{2}\right) f_{0}^{(1)}(z)
$$

It follows (2.2) and (2.3) that the function $F$ is analytic in $\mathbb{C}_{+}$, continuous up to the real axis, and

$$
F(z+4 \pi)=F(z)
$$


We denote the set of eigenvalues and spectral singularities of $L$ by $\sigma_{d}(L)$ and $\sigma_{\mathrm{ss}}(L)$, respectively. From the definition of the eigenvalues and spectral singularities we have [10]

$$
\begin{gathered}
\sigma_{d}=\left\{\lambda: \lambda=2 \sin \frac{z}{2}, z \in P_{0}, F(z)=0\right\}, \\
\sigma_{\mathrm{ss}}=\left\{\lambda: \lambda=2 \sin \frac{z}{2}, z \in[0,4 \pi], F(z)=0\right\},
\end{gathered}
$$

where $P_{0}:=\{z: z \in \mathbb{C}, z=x+i y, 0 \leq x \leq 4 \pi, y>0\}$. The finiteness of the multiplicities of eigenvalues and spectral singularities has been proven in [10]. Using (2.2), (2.3), and (2.8) we obtain

$$
\begin{aligned}
F(z)= & \left\{\gamma_{0}+\gamma_{1}\left[(-i)\left(e^{i(z / 2)}-e^{-i(z / 2)}\right)\right]\right\} f_{1}^{(2)}(z) \\
& +\left\{\beta_{0}+\beta_{1}\left[(-i)\left(e^{i(z / 2)}-e^{-i(z / 2)}\right)\right]\right\} f_{0}^{(1)}(z) \\
= & i \alpha_{0}^{11} \beta_{1}+\left(\gamma_{1} \alpha_{1}^{22}+\alpha_{0}^{11} \beta_{0}\right) e^{i(z / 2)}+i\left(-\gamma_{0} \alpha_{1}^{22}+\gamma_{1} \alpha_{1}^{22}-\alpha_{0}^{11} \beta_{1}\right) e^{i z} \\
& +\left(\gamma_{0} \alpha_{1}^{21}-\gamma_{1} \alpha_{1}^{22}\right) e^{i(3 z / 2)}-i \gamma_{1} \alpha_{1}^{21} e^{2 i z} \\
& +\sum_{m=1}^{\infty} \alpha_{0}^{11} \beta_{1} A_{0 m}^{12} e^{i(m-(1 / 2)) z}+i \sum_{m=1}^{\infty}\left(-\alpha_{0}^{11} \beta_{0} A_{0 m}^{12}+\alpha_{0}^{11} \beta_{1} A_{0 m}^{11}\right) e^{i m z} \\
& +\sum_{m=1}^{\infty}\left(\gamma_{1} \alpha_{1}^{21} A_{1 m}^{12}+\gamma_{1} \alpha_{1}^{22} A_{1 m}^{22}+\alpha_{0}^{11} \beta_{0} A_{0 m}^{11}-\alpha_{0}^{11} \beta_{1} A_{0 m}^{12}\right) e^{i(m+(1 / 2)) z} \\
& +i \sum_{m=1}^{\infty}\left(-\gamma_{0} \alpha_{1}^{21} A_{1 m}^{12}-\gamma_{0} \alpha_{1}^{22} A_{1 m}^{22}+\gamma_{1} \alpha_{1}^{21} A_{1 m}^{11}+\gamma_{1} \alpha_{1}^{22} A_{1 m}^{21}\right. \\
& \left.\quad-\alpha_{0}^{11} \beta_{1} A_{0 m}^{11}\right) e^{i(m+1) z} \\
& +\sum_{m=1}^{\infty}\left(\gamma_{0} \alpha_{1}^{21} A_{1 m}^{11}+\gamma_{0} \alpha_{1}^{22} A_{1 m}^{21}-\gamma_{1} \alpha_{1}^{21} A_{1 m}^{12}-\gamma_{1} \alpha_{1}^{22} A_{1 m}^{22}\right) e^{i(m+(3 / 2)) z} \\
& +i \sum_{m=1}^{\infty}\left(-\gamma_{1} \alpha_{1}^{21} A_{1 m}^{11}-\gamma_{1} \alpha_{1}^{22} A_{1 m}^{21}\right) e^{i(m+2) z} . \\
& +i \sum_{m=1}^{\infty}\left(-\gamma_{1} \alpha_{1}^{21} A_{1 m}^{11}-\gamma_{1} \alpha_{1}^{22} A_{1 m}^{21}\right) e^{i(m+2) z} .
\end{aligned}
$$

Definition 2.1. The multiplicity of a zero of $F$ in $P:=P_{0} \cup[0,4 \pi]$ is called the multiplicity of the corresponding eigenvalue or spectral singularity of the BVP (1.2), (1.3).

\section{Principal Functions}

In this section we also assume that (2.1) holds.

Let $\lambda_{1}, \lambda_{2}, \ldots, \lambda_{k}$ and $\lambda_{k+1}, \lambda_{k+2}, \ldots, \lambda_{v}$ denote the zeros of $F$ in $P_{0}$ and $[0,4 \pi]$ with multiplicities $m_{1}, m_{2}, \ldots, m_{k}$ and $m_{k+1}, m_{k+2}, \ldots, m_{v}$, respectively. 
Let us define $\ell:=\left(\begin{array}{c}\tilde{\ell} \\ \hat{\ell}\end{array}\right)$ where

$$
\begin{aligned}
& (\tilde{e} y)_{n}=a_{n+1} y_{n+1}^{(2)}+b_{n} y_{n}^{(2)}+p_{n} y_{n}^{(1)}, \quad n \in \mathbb{N}, \\
& (\hat{\ell} y)_{n}=a_{n-1} y_{n-1}^{(1)}+b_{n} y_{n}^{(1)}+q_{n} y_{n}^{(2)}, \quad n \in \mathbb{N} .
\end{aligned}
$$

Definition 3.1. Let $\lambda=\lambda_{0}$ be an eigenvalue of $L$. If the vectors $y_{n}, d /\left(d \lambda y_{n}\right), d^{2} / d \lambda^{2} y_{n}, \ldots$, $d^{v} / d \lambda^{v} y_{n}$

$$
\frac{d^{j}}{d \lambda^{j}} y:=\left\{\frac{d^{j}}{d \lambda^{j}} y_{n}\right\}_{n \in \mathbb{N}}, \quad(j=0,1, \ldots, v ; n \in \mathbb{N})
$$

satisfy the equations

$$
\begin{aligned}
&(\ell y)_{n}-\lambda_{0} y_{n}=0, \\
&\left(\ell\left(\frac{d^{j}}{d \lambda^{j}} y\right)\right)_{n}-\lambda_{0} \frac{d^{j}}{d \lambda^{j}} y_{n}-\frac{d^{j-1}}{d \lambda^{j-1}} y_{n}=0, \quad j=1,2, \ldots, v, n \in \mathbb{N},
\end{aligned}
$$

then the vector $y_{n}$ is called the eigenvector corresponding to the eigenvalue $\lambda=\lambda_{0}$ of $L$. The vectors $(d / d \lambda) y_{n},\left(d^{2} / d \lambda^{2}\right) y_{n}, \ldots,\left(d^{v} / d \lambda^{v}\right) y_{n}$ are called the associated vectors corresponding to $\lambda=\lambda_{0}$. The eigenvector and the associated vectors corresponding to $\lambda=\lambda_{0}$ are called the principal vectors of the eigenvalue $\lambda=\lambda_{0}$. The principal vectors of the spectral singularities of $L$ are defined similarly.

We define the vectors

$$
\begin{gathered}
\frac{d^{j}}{d \lambda^{j}} V_{n}\left(\lambda_{i}\right)=\left(\begin{array}{l}
\frac{1}{j !}\left\{\frac{d^{j}}{d \lambda^{j}} E_{n}^{(1)}(\lambda)\right\}_{\lambda=\lambda_{i}} \\
\frac{1}{j !}\left\{\frac{d^{j}}{d \lambda^{j}} E_{n}^{(2)}(\lambda)\right\}_{\lambda=\lambda_{i}}
\end{array}\right), \\
n \in \mathbb{N}, \quad j=0,1, \ldots, m_{i}-1, \quad i=1,2, \ldots, k, k+1, \ldots, v,
\end{gathered}
$$

where $\lambda=2 \sin (z / 2)$ and

$$
E_{n}(\lambda)=\left(\begin{array}{l}
E_{n}^{(1)}(\lambda) \\
E_{n}^{(2)}(\lambda)
\end{array}\right):=f_{n}\left(2 \arcsin \frac{\lambda}{2}\right)=\left(\begin{array}{c}
f_{n}^{(1)}\left(2 \arcsin \frac{\lambda}{2}\right) \\
f_{n}^{(2)}\left(2 \arcsin \frac{\lambda}{2}\right)
\end{array}\right) .
$$


If

$$
y(\lambda)=\left\{y_{n}(\lambda)\right\}:=\left(\begin{array}{l}
y_{n}^{(1)}(\lambda) \\
y_{n}^{(2)}(\lambda)
\end{array}\right)_{n \in \mathbb{N}}
$$

is a solution of (1.2), then

$$
\frac{d^{j}}{d \lambda^{j}} y(\lambda)=\left\{\left(\frac{d^{j}}{d \lambda^{j}}\right) y_{n}(\lambda)\right\}_{n \in \mathbb{N}}:=\left\{\begin{array}{l}
\left(\frac{d^{j}}{d \lambda^{j}}\right) y_{n}^{(1)}(\lambda) \\
\left(\frac{d^{j}}{d \lambda^{j}}\right) y_{n}^{(2)}(\lambda)
\end{array}\right\}
$$

satisfies

$$
\begin{gathered}
\left(\begin{array}{c}
a_{n-1} \frac{d^{j}}{d \lambda^{j}} y_{n+1}^{(2)}(\lambda)+b_{n} \frac{d^{j}}{d \lambda^{j}} y_{n}^{(2)}(\lambda)+p_{n} \frac{d^{j}}{d \lambda^{j}} y_{n}^{(1)}(\lambda) \\
a_{n-1} \frac{d^{j}}{d \lambda^{j}} y_{n-1}^{(1)}(\lambda)+b_{n} \frac{d^{j}}{d \lambda^{j}} y_{n}^{(1)}(\lambda)+q_{n} \frac{d^{j}}{d \lambda^{j}} y_{n}^{(2)}(\lambda)
\end{array}\right) \\
=\left(\begin{array}{c}
\lambda \frac{d^{j}}{d \lambda^{j}} y_{n}^{(1)}(\lambda)+j \frac{d^{j-1}}{d \lambda^{j-1}} y_{n}^{(1)}(\lambda) \\
\lambda \frac{d^{j}}{d \lambda^{j}} y_{n}^{(2)}(\lambda)+j \frac{d^{j-1}}{d \lambda^{j-1}} y_{n}^{(2)}(\lambda)
\end{array}\right)
\end{gathered}
$$

From (3.4) and (3.8) we get that

$$
\begin{gathered}
\left(\ell V\left(\lambda_{i}\right)\right)_{n}-\lambda_{0} V_{n}\left(\lambda_{i}\right)=0 \\
\left(\ell\left(\frac{d^{j}}{d \lambda^{j}} V\left(\lambda_{i}\right)\right)\right)_{n}-\lambda_{0} \frac{d^{j}}{d \lambda^{j}} V_{n}\left(\lambda_{i}\right)-\frac{d^{j-1}}{d \lambda^{j-1}} V_{n}\left(\lambda_{i}\right)=0 \\
n \in \mathbb{N}, \quad j=1,2, \ldots, m_{i}-1, \quad i=1,2, \ldots, v .
\end{gathered}
$$

The vectors $d^{j} / d \lambda^{j} V_{n}\left(\lambda_{i}\right), j=0,1,2, \ldots, m_{i}-1, i=1,2, \ldots, k$ and $d^{j} / d \lambda^{j} V_{n}\left(\lambda_{i}\right), j=0,1,2, \ldots$, $m_{i}-1, i=k+1, k+2, \ldots, v$ are the principal vectors of eigenvalues and spectral singularities of $L$, respectively.

\section{Theorem 3.2.}

$$
\begin{gathered}
\frac{d^{j}}{d \lambda^{j}} V_{n}\left(\lambda_{i}\right) \in \ell_{2}\left(\mathbb{N}, \mathbb{C}^{2}\right), \quad j=0,1,2, \ldots, m_{i}-1, \quad i=1,2, \ldots, k, \\
\frac{d^{j}}{d \lambda^{j}} V_{n}\left(\lambda_{i}\right) \notin \ell_{2}\left(\mathbb{N}, \mathbb{C}^{2}\right), \quad j=0,1,2, \ldots, m_{i}-1, \quad i=k+1, k+2, \ldots, v .
\end{gathered}
$$


Proof. Using (3.5) we get that

$$
\begin{aligned}
& \left\{\frac{d^{j}}{d \lambda^{j}} E_{n}^{(1)}(\lambda)\right\}_{\lambda=\lambda_{i}}=\sum_{t=0}^{j} C_{t}\left\{\frac{d^{t}}{d \lambda^{t}} f_{n}^{(1)}(z)\right\}_{z=z_{i}}, \quad n \in \mathbb{N}, \\
& \left\{\frac{d^{j}}{d \lambda^{j}} E_{n}^{(2)}(\lambda)\right\}_{\lambda=\lambda_{i}}=\sum_{t=0}^{j} D_{t}\left\{\frac{d^{t}}{d \lambda^{t}} f_{n}^{(2)}(z)\right\}_{z=z_{i}}, \quad n \in \mathbb{N},
\end{aligned}
$$

where $\lambda_{i}=2 \sin z_{i} / 2, z_{i} \in P=P_{0} \cup[0,4], i=1,2, \ldots, k$ and $C_{t}, D_{t}$ are constant depending on $\lambda$. From (2.2) we obtain that

$$
\begin{aligned}
&\left\{\frac{d^{t}}{d \lambda^{t}} f_{n}^{(1)}(z)\right\}_{z=z_{i}}= \alpha_{n}^{11} i^{t}\left(n+\frac{1}{2}\right)^{t} e^{i z_{i}(n+(1 / 2))} \\
&+\sum_{m=1}^{\infty} \alpha_{n}^{11}\left\{A_{n m}^{11} i^{t}\left(m+n+\frac{1}{2}\right)^{t} e^{i(m+n+(1 / 2)) z_{i}}\right. \\
&\left.-A_{n m}^{12} i^{t+1}(m+n)^{t} e^{i(m+n) z_{i}}\right\}, \\
&\left\{\frac{d^{t}}{d \lambda^{t}} f_{n}^{(2)}(z)\right\}_{z=z_{i}}= \alpha_{n}^{21} i^{t}\left(n+\frac{1}{2}\right)^{t} e^{i z_{i}(n+(1 / 2))}-i(i n)^{t} \alpha_{n}^{22} e^{i n z_{i}} \\
&+\sum_{m=1}^{\infty} \alpha_{n}^{21}\left\{A_{n m}^{11} i^{t}\left(m+n+\frac{1}{2}\right)^{t} e^{i(m+n+(1 / 2)) z_{i}}\right. \\
&\left.-A_{n m}^{12} i^{t+1}(m+n)^{t} e^{i(m+n) z_{i}}\right\} \\
&+\sum_{m=1}^{\infty} \alpha_{n}^{22}\left\{A_{n m}^{21} i^{t}\left(m+n+\frac{1}{2}\right)^{t} e^{i(m+n+(1 / 2)) z_{i}}\right. \\
&\left.-A_{n m}^{22} i^{t+1}(m+n)^{t} e^{i(m+n) z_{i}}\right\} .
\end{aligned}
$$

For the principal vectors $\left(d^{j} / d \lambda^{j}\right) V_{n}\left(\lambda_{i}\right)=\left\{\left(d^{j} / d \lambda^{j}\right) V_{n}\left(\lambda_{i}\right)\right\}_{n \in \mathbb{N}}, j=0,1, \ldots, m_{i}-1, i=1,2$, $\ldots, k$ corresponding to the eigenvalues of $L$ we get

$$
\begin{gathered}
\frac{1}{j !}\left\{\frac{d^{j}}{d \lambda^{j}} E_{n}^{(1)}(\lambda)\right\}_{\lambda=\lambda_{i}}=\frac{1^{j}}{j !} \sum_{t=0}^{j} C_{t}\left\{\frac{d^{t}}{d \lambda^{t}} f_{n}^{(1)}\left(z_{i}\right)\right\}, \\
j=0,1, \ldots, m_{i}-1, \quad i=1,2, \ldots, k, \\
\frac{1}{j !}\left\{\frac{d^{j}}{d \lambda^{j}} E_{n}^{(2)}(\lambda)\right\}_{\lambda=\lambda_{i}}=\frac{1^{j}}{j !} \sum_{t=0}^{j} D_{t}\left\{\frac{d^{t}}{d \lambda^{t}} f_{n}^{(2)}\left(z_{i}\right)\right\}, \\
j=0,1, \ldots, m_{i}-1, \quad i=1,2, \ldots, k .
\end{gathered}
$$


Since $\operatorname{Im} \lambda_{i}>0$ for $i=1,2, \ldots, k$ from (3.14) we obtain that

$$
\begin{aligned}
\left\|\frac{d^{j}}{d \lambda^{j}} V_{n}\right\|^{2} & =\sum_{n=1}^{\infty}\left(\left|\frac{1}{j !}\left\{\frac{d^{j}}{d \lambda^{j}} E_{n}^{(1)}(\lambda)\right\}_{\lambda=\lambda_{i}}\right|^{2}+\left|\frac{1}{j !}\left\{\frac{d^{j}}{d \lambda^{j}} E_{n}^{(2)}(\lambda)\right\}_{\lambda=\lambda_{i}}\right|^{2}\right) \\
& =\left(\frac{1}{j !}\right)^{2} \sum_{n=1}^{\infty}\left(\left|\sum_{t=0}^{j} C_{t}\left\{\frac{d^{t}}{d \lambda^{t}} f_{n}^{(1)}\left(z_{i}\right)\right\}\right|^{2}+\left|\sum_{t=0}^{j} D_{t}\left\{\frac{d^{t}}{d \lambda^{t}} f_{n}^{(2)}\left(z_{i}\right)\right\}\right|^{2}\right) \\
& \leq\left(\frac{1}{j !}\right)^{2} \sum_{n=1}^{\infty}\left\{\left(\sum_{t=0}^{j}\left|C_{t}\left\{\frac{d^{t}}{d \lambda^{t}} f_{n}^{(1)}\left(z_{i}\right)\right\}\right|^{2}\right)+\left(\sum_{t=0}^{j}\left|D_{t}\left\{\frac{d^{t}}{d \lambda^{t}} f_{n}^{(2)}\left(z_{i}\right)\right\}\right|^{2}\right)\right\} \\
& \leq\left(\frac{1}{j !}\right)^{2}\left(\sum_{n=1}^{\infty} \sum_{t=0}^{j} \max \left\{\left|C_{t}\right|,\left|D_{t}\right|\right\}\left(\left|\left\{\frac{d^{t}}{d \lambda^{t}} f_{n}^{(1)}\left(z_{i}\right)\right\}\right|+\left|\left\{\frac{d^{t}}{d \lambda^{t}} f_{n}^{(2)}\left(z_{i}\right)\right\}\right|\right)\right)^{2},
\end{aligned}
$$

or

$$
\begin{aligned}
\left\|\frac{d^{j}}{d \lambda^{j}} V_{n}\right\|^{2} & \\
\leq\left(\frac{1}{j !}\right)^{2}\left\{\sum _ { n = 1 } ^ { \infty } \left[\sum_{t=0}^{j} \max \left\{\left|C_{t}\right|,\left|D_{t}\right|\right\}\right.\right. & \\
& \times\left\{\left(\left|\alpha_{n}^{11}\right|+\left|\alpha_{n}^{21}\right|\right)\left(\left|n+\frac{1}{2}\right|^{t} e^{-(n+(1 / 2)) \operatorname{Im} z_{i}}\right)+\left|\alpha_{n}^{22}\right||n|^{t} e^{-n \operatorname{Im} z_{i}}\right\} \\
+ & \sum_{t=0}^{j} \max \left\{\left|C_{t}\right|,\left|D_{t}\right|\right\}\left\{\sum_{m=1}^{\infty}\left(\left|\alpha_{n}^{11}\right|+\left|\alpha_{n}^{21}\right|\right)\left(\left|A_{n m}^{11}\right|\left|m+n+\frac{1}{2}\right|^{t} e^{-(m+n+(1 / 2)) \operatorname{Im} z_{i}}\right)\right. \\
& \left.+\left|A_{n m}^{12}\right||m+n|^{t} e^{-(m+n) \operatorname{Im} z_{i}}\right\} \\
+ & \sum_{t=0}^{j}\left|D_{t}\right|\left\{\sum _ { m = 1 } ^ { \infty } | \alpha _ { n } ^ { 2 2 } | \left(\left|A_{n m}^{21}\right|\left|m+n+\frac{1}{2}\right|^{t} e^{-(m+n+(1 / 2)) \operatorname{Im} z_{i}}+\left|A_{n m}^{22}\right||m+n|^{t}\right.\right. \\
& \left.\left.\left.\left.\times e^{-(m+n) \operatorname{Im} z_{i}}\right)\right\}\right]\right\}^{2} .
\end{aligned}
$$

From (3.16),

$$
\begin{gathered}
\left(\frac{1}{j !}\right)^{2} \sum_{n=1}^{\infty} \sum_{t=0}^{j} \max \left\{\left|C_{t}\right|,\left|D_{t}\right|\right\}\left\{\begin{array}{c}
\left(\left|\alpha_{n}^{11}\right|+\left|\alpha_{n}^{21}\right|\right)\left(\left|n+\frac{1}{2}\right|^{t} e^{-(n+(1 / 2)) \operatorname{Im} z_{i}}\right) \\
+\left|\alpha_{n}^{22}\right||n|^{t} e^{-n \operatorname{Im} z_{i}}
\end{array}\right\} \\
\leq \frac{A}{(j !)^{2}} \sum_{n=1}^{\infty}\left(1+\left(n+\frac{1}{2}\right)+\left(n+\frac{1}{2}\right)^{2}+\cdots+\left(n+\frac{1}{2}\right)^{j}\right) e^{-(n+(1 / 2)) \operatorname{Im} z_{i}}
\end{gathered}
$$




$$
\begin{aligned}
& +\left(1+n+n^{2}+\cdots+n^{j}\right) e^{-n \operatorname{Im} z_{i}} \\
\leq & \frac{A(j+1)^{2}}{(j !)^{2}} \sum_{n=1}^{\infty}\left[\left(n+\frac{1}{2}\right)^{j} e^{-(n+(1 / 2)) \operatorname{Im} z_{i}}+n^{j} e^{-n \operatorname{Im} z_{i}}\right]<\infty .
\end{aligned}
$$

Holds, where

$$
A=\max \left\{\left|C_{t}\right|,\left|D_{t}\right|\right\} \max \left\{\left(\left|\alpha_{n}^{11}\right|+\left|\alpha_{n}^{21}\right|\right),\left|\alpha_{n}^{22}\right|\right\}
$$

Now we define the function

$$
\begin{aligned}
g_{n}(z)= & \sum_{t=0}^{j} \max \left\{\left|C_{t}\right|,\left|D_{t}\right|\right\}\left\{\sum _ { m = 1 } ^ { \infty } ( | \alpha _ { n } ^ { 1 1 } | + | \alpha _ { n } ^ { 2 1 } | ) \left(\left|A_{n m}^{11}\right|\left|m+n+\frac{1}{2}\right|^{t} e^{-(m+n+(1 / 2)) \operatorname{Im} z_{i}}\right.\right. \\
& \left.\left.+\left|A_{n m}^{12}\right||m+n|^{t} e^{-(m+n) \operatorname{Im} z_{i}}\right)\right\} \\
& +\sum_{t=0}^{j}\left|D_{t}\right|\left\{\sum _ { m = 1 } ^ { \infty } | \alpha _ { n } ^ { 2 2 } | \left(\left|A_{n m}^{21}\right||| m+n+\left.\frac{1}{2}\right|^{t} e^{-(m+n+(1 / 2)) \operatorname{Im} z_{i}}\right.\right. \\
& \left.\left.+\left|A_{n m}^{22}\right||m+n|^{t} e^{-(m+n) \operatorname{Im} z_{i}}\right)\right\} .
\end{aligned}
$$

So we get

$$
\begin{array}{r}
\left(\frac{1}{j !}\right)^{2} \sum_{n=1}^{\infty}\left[\sum _ { t = 0 } ^ { j } \operatorname { m a x } \{ | C _ { t } | , | D _ { t } | \} \left\{\sum _ { m = 1 } ^ { \infty } ( | \alpha _ { n } ^ { 1 1 } | + | \alpha _ { n } ^ { 2 1 } | ) \left(\left|A_{n m}^{11}\right|\left|m+n+\frac{1}{2}\right|^{t} e^{-(m+n+(1 / 2)) \operatorname{Im} z_{i}}\right.\right.\right. \\
\left.+\left|A_{n m}^{12}\right||m+n|^{t} e^{\left.-(m+n) \operatorname{Im} z_{i}\right)}\right\} \\
+\sum_{t=0}^{j}\left|D_{t}\right|\left\{\sum _ { m = 1 } ^ { \infty } | \alpha _ { n } ^ { 2 2 } | \left(\left|A_{n m}^{21}\right|\left|m+n+\frac{1}{2}\right|^{t} e^{-(m+n+(1 / 2)) \operatorname{Im} z_{i}}\right.\right. \\
+\left(\frac{1}{j !}\right)^{2} \sum_{n=1}^{\infty} g_{n}(z) .
\end{array}
$$


Using the boundness of $A_{n m}^{i j}$ and $\alpha_{n}^{i j}, i, j=1,2$ we obtain that

$$
\begin{gathered}
g_{n}(z) \leq \max \left\{\left|C_{t}\right|,\left|D_{t}\right|\right\} M \sum_{t=0}^{j} \sum_{m=1}^{\infty}\left\{\left|m+n+\frac{1}{2}\right|^{t} e^{-(m+n+(1 / 2)) \operatorname{Im} z_{i}}\right. \\
\left.+|m+n|^{t} e^{-(m+n) \operatorname{Im} z_{i}}\right\},
\end{gathered}
$$

where

$$
\begin{aligned}
& M=\max \left\{\left(\left|\alpha_{n}^{11}\right|+\left|\alpha_{n}^{21}\right|\right)\left|A_{n m}^{11}\right|,\left|\alpha_{n}^{22}\right|\left|A_{n m}^{21}\right|\right. \\
&\left.\left(\left|\alpha_{n}^{11}\right|+\left|\alpha_{n}^{21}\right|\right)\left|A_{n m}^{12}\right|,\left|\alpha_{n}^{22}\right|\left|A_{n m}^{22}\right|\right\} .
\end{aligned}
$$

If we take $\max \left\{\left|C_{t}\right|,\left|D_{t}\right|\right\} M=N$, we can write

$$
\begin{aligned}
& g_{n}(z) \leq N \sum_{t=0}^{j} e^{-n \operatorname{Im} z_{i}} \sum_{m=1}^{\infty}\left\{\left(m+n+\frac{1}{2}\right)^{t} e^{-m \operatorname{Im} z_{i}}+(m+n)^{t} e^{-m \operatorname{Im} z_{i}}\right\} \\
&= N e^{-n \operatorname{Im} z_{i}}\left\{\sum_{m=1}^{\infty} 2 e^{-m \operatorname{Im} z_{i}}+\sum_{m=1}^{\infty} e^{-m \operatorname{Im} z_{i}}\left(\left(m+n+\frac{1}{2}\right)+(m+n)\right)+\cdots\right. \\
&\left.+\sum_{m=1}^{\infty} e^{-m \operatorname{Im} z_{i}}\left(\left(m+n+\frac{1}{2}\right)^{j}+(m+n)^{j}\right)\right\} \\
& \leq N e^{-n \operatorname{Im} z_{i}} \sum_{m=1}^{\infty} \sum_{t=0}^{j} e^{-m \operatorname{Im} z_{i}}\left(\left(m+n+\frac{1}{2}\right)^{t}+(m+n)^{t}\right) \\
& \leq B e^{-n \operatorname{Im} z_{i}},
\end{aligned}
$$

where

$$
B=A \sum_{m=1}^{\infty} \sum_{t=0}^{j} e^{-m \operatorname{Im} z_{i}}\left(\left(m+n+\frac{1}{2}\right)^{t}+(m+n)^{t}\right) .
$$

Therefore we have

$$
\left(\frac{1}{j !}\right)^{2} \sum_{n=1}^{\infty} g_{n}(z) \leq\left(\frac{1}{j !}\right)^{2} \sum_{n=1}^{\infty} B e^{-n \operatorname{Im} z_{i}}<\infty
$$

From (3.17) and (3.25) $d^{j} / d \lambda^{j} V_{n}\left(\lambda_{i}\right) \in \ell_{2}\left(\mathbb{N}, \mathbb{C}^{2}\right), j=0,1, \ldots, m_{i}-1, i=1,2, \ldots, k$. 
On the other hand, since $\operatorname{Im} z_{i}=0$ for $j=0,1, \ldots, m_{i}-1 ; i=k+1, k+2, \ldots, v$ using (3.12) we find that

$$
\sum_{n=1}^{\infty}\left|\alpha_{n}^{11} i^{t}\left(n+\frac{1}{2}\right)^{t} e^{i z_{i}(n+(1 / 2))}\right|^{2}=\infty
$$

but the other terms in (3.12) belongs $\ell_{2}\left(\mathbb{N}, \mathbb{C}^{2}\right)$, so $d^{j} /\left(d \lambda^{j}\right) E_{n}^{(1)}(\lambda) \notin \ell_{2}\left(\mathbb{N}, \mathbb{C}^{2}\right)$. Similarly from (3.13) we get $d^{j} /\left(d \lambda^{j}\right) E_{n}^{(2)}(\lambda) \notin \ell_{2}\left(\mathbb{N}, \mathbb{C}^{2}\right)$, then we obtain that $d^{j} /\left(d \lambda^{j}\right) V_{n}\left(\lambda_{i}\right) \notin \ell_{2}\left(\mathbb{N}, \mathbb{C}^{2}\right)$, $j=0,1, \ldots, m_{i}-1 ; i=k+1, k+2, \ldots, v$.

Let us introduce Hilbert space $j=0,1,2, \ldots$

$$
H_{-j}(\mathbb{N})=\left\{y=\left(\begin{array}{c}
y_{n}^{(1)} \\
y_{n}^{(2)}
\end{array}\right): \sum_{n \in \mathbb{N}}(1+|n|)^{-2 j}\left(\left|y_{n}^{(1)}\right|^{2}+\left|y_{n}^{(2)}\right|^{2}\right)<\infty\right\},
$$

with

$$
\|y\|_{-j}^{2}=\sum_{n \in \mathbb{N}}(1+|n|)^{-2 j}\left(\left|y_{n}^{(1)}\right|^{2}+\left|y_{n}^{(2)}\right|^{2}\right)
$$

Theorem 3.3. $d^{j} /\left(d \lambda^{j}\right) V_{n}\left(\lambda_{i}\right) \in H_{-(j+1)}(\mathbb{N}), j=0,1,2, \ldots, m_{i}-1, i=k+1, k+2, \ldots, v$.

Proof. Using (3.4), (3.14) we have

$$
\begin{aligned}
\sum_{n \in \mathbb{N}}(1 & +|n|)^{-2(j+1)}\left(\left|\frac{1}{j !}\left\{\frac{d^{j}}{d \lambda^{j}} E_{n}^{(1)}(\lambda)\right\}_{\lambda=\lambda_{i}}\right|^{2}+\left.\left|\frac{1}{j !}\left\{\frac{d^{j}}{d \lambda^{j}} E_{n}^{(2)}(\lambda)\right\}_{\lambda=\lambda_{i}}\right|\right|^{2}\right) \\
& =\sum_{n \in \mathbb{N}} \frac{(1+|n|)^{-2(j+1)}}{(j !)^{2}}\left\{\left|\sum_{t=0}^{j} C_{t}\left\{\frac{d^{t}}{d \lambda^{t}} f_{n}^{(1)}\left(z_{i}\right)\right\}\right|^{2}+\left|\sum_{t=0}^{j} D_{t}\left\{\frac{d^{t}}{d \lambda^{t}} f_{n}^{(2)}\left(z_{i}\right)\right\}\right|^{2}\right\} \\
& \leq \frac{1}{(j !)^{2}} \sum_{n=1}^{\infty}(1+|n|)^{-2(j+1)}\left\{\left(\sum_{t=0}^{j}\left|C_{t}\left\{\frac{d^{t}}{d \lambda^{t}} f_{n}^{(1)}\left(z_{i}\right)\right\}\right|\right)^{2}+\left(\sum_{t=0}^{j}\left|D_{t}\left\{\frac{d^{t}}{d \lambda^{t}} f_{n}^{(2)}\left(z_{i}\right)\right\}\right|\right)^{2}\right\},
\end{aligned}
$$

for $j=0,1,2, \ldots, m_{i}-1, i=k+1, k+2, v$. Since $\operatorname{Im} z_{i}=0$, using (3.29) we obtain

$$
\begin{aligned}
& \sum_{n \in \mathbb{N}}(1+|n|)^{-2(j+1)} \frac{1}{(j !)^{2}}\left(\sum_{t=0}^{j}\left|C_{t}\left\{\frac{d^{t}}{d \lambda^{t}} f_{n}^{(1)}\left(z_{i}\right)\right\}\right|\right)^{2} \\
&=\frac{1}{(j !)^{2}} \sum_{n=1}^{\infty}\left\{\sum_{t=0}^{j}(1+|n|)^{-(j+1)}\left(n+\frac{1}{2}\right)^{t}\left|\alpha_{n}^{11}\right|\left|C_{t}\right|\right. \\
&\left.\quad+\sum_{t=0}^{j}\left|C_{t}\right|\left|\alpha_{n}^{11}\right|(1+|n|)^{-(j+1)} \sum_{m=1}^{\infty}\left|A_{n m}^{11}\right|\left(m+n+\frac{1}{2}\right)^{t}+\left|A_{n m}^{12}\right|(m+n)^{t}\right\}^{2}
\end{aligned}
$$


Abstract and Applied Analysis

$$
\begin{aligned}
=\frac{1}{(j !)^{2}} \sum_{n=1}^{\infty}\{ & \left(\sum_{t=0}^{j}(1+|n|)^{-(j+1)}\left(n+\frac{1}{2}\right)^{t}\left|\alpha_{n}^{11}\right|\left|C_{t}\right|\right)^{2} \\
& +2(1+|n|)^{-2(j+1)}\left|\alpha_{n}^{11}\right|^{2}\left[\sum_{t=0}^{j}\left(n+\frac{1}{2}\right)^{t}\left|C_{t}\right|\right] \\
& \times\left[\sum_{t=0}^{j}\left|C_{t}\right| \sum_{m=1}^{\infty}\left|A_{n m}^{11}\right|\left(m+n+\frac{1}{2}\right)^{t}+\left|A_{n m}^{12}\right|(m+n)^{t}\right] \\
& \left.+\left(\sum_{t=0}^{j}\left|C_{t}\right|(1+|n|)^{-(j+1)}\left|\alpha_{n}^{11}\right| \sum_{m=1}^{\infty}\left|A_{n m}^{11}\right|\left(m+n+\frac{1}{2}\right)^{t}+\left|A_{n m}^{12}\right|(m+n)^{t}\right)^{2}\right\} .
\end{aligned}
$$

Using (3.30), (2.1), and (2.7) we first obtain that

$$
\begin{gathered}
\left(\sum_{t=0}^{j}\left|C_{t}\right|\left|\alpha_{n}^{11}\right|(1+|n|)^{-(j+1)} \sum_{m=1}^{\infty}\left(\left|A_{n m}^{11}\right|\left(m+\mathrm{n}+\frac{1}{2}\right)^{t}+\left|A_{n m}^{12}\right|(m+n)^{t}\right)\right)^{2} \\
\leq 4\left(\sum_{t=0}^{j}\left|C_{t}\right|\left|\alpha_{n}^{11}\right|_{m=1}^{\infty}(1+|n|)^{-(j+1)}\left(m+n+\frac{1}{2}\right)^{t} C\right. \\
\left.\times \sum_{j=n+|m / 2|}^{\infty}\left(\left|1-a_{j}\right|+\left|1+b_{j}\right|+\left|p_{j}\right|+\left|q_{j}\right|\right) e^{-\varepsilon j^{\delta}} e^{\varepsilon j^{\delta}}\right)^{2} \\
\leq 4\left\{\sum_{t=0}^{j}\left|\alpha_{n}^{11}\right| \sum_{m=1}^{\infty}(1+|n|)^{-(j+1)}\left(m+n+\frac{1}{2}\right)^{t} C \exp \left(-\varepsilon\left(\frac{n+m}{4}\right)^{\delta}\right)\right. \\
\left.\quad \times \sum_{j=n+\lceil m / 2 \mid}^{\infty} e^{\varepsilon j^{\delta}}\left(\left|1-a_{j}\right|+\left|1+b_{j}\right|+\left|p_{j}\right|+\left|q_{j}\right|\right)\right\}^{2} \\
\leq C_{1}\left(\sum_{t=0}^{j}(1+|n|)^{-(j+1)} \sum_{m=1}^{\infty}\left(m+n+\frac{1}{2}\right)^{t} \exp \left(-\varepsilon\left(\frac{n+m}{4}\right)^{\delta}\right)\right)^{2} \\
\leq C_{1}\left(\sum_{t=0}^{j}(1+|n|)^{-(j+1)} \sum_{m=1}^{\infty}\left(m+n+\frac{1}{2}\right)^{t} \exp \left(-\varepsilon\left(\frac{n+m}{4}\right)^{1 / 2}\right)\right)^{2} \\
\leq C_{1}\left(\sum_{t=0}^{j}(1+|n|)^{-(j+1)} \sum_{m=1}^{\infty}\left(m+n+\frac{1}{2}\right)^{t} \exp \left(-\varepsilon \frac{\sqrt{2}}{4}\left(\frac{1}{n} \frac{1}{2}+m \frac{1}{2}\right)\right)^{2}\right.
\end{gathered}
$$




$$
\begin{aligned}
& =C_{1}(1+|n|)^{-2(j+1)} \exp \left(-\varepsilon \frac{\sqrt{2}}{4} n^{1 / 2}\right)\left(\sum_{t=0}^{j} \sum_{m=1}^{\infty}\left(m+n+\frac{1}{2}\right)^{t} \exp \left(-\varepsilon \frac{\sqrt{2}}{4} m^{1 / 2}\right)\right)^{2} \\
& =G \exp \left(-\varepsilon \frac{\sqrt{2}}{4} n^{\frac{1}{2}}\right)(1+|n|)^{-2(j+1)}
\end{aligned}
$$

where

$$
\begin{gathered}
C_{1}=\left(2 C\left|\alpha_{n}^{11}\right| \sum_{j=n+\lceil m / 2\rceil}^{\infty} e^{\varepsilon j^{\delta}}\left(\left|1-a_{j}\right|+\left|1+b_{j}\right|+\left|p_{j}\right|+\left|q_{j}\right|\right)\right)^{2}, \\
G=C_{1}\left(\sum_{t=0}^{j} \sum_{m=1}^{\infty}\left(m+n+\frac{1}{2}\right)^{t} \exp \left(-\varepsilon \frac{\sqrt{2}}{4} m^{1 / 2}\right)\right)^{2} .
\end{gathered}
$$

So we get from (3.31)

$$
\begin{aligned}
& \sum_{n=1}^{\infty}\left(\sum_{t=0}^{j}\left|C_{t}\right|(1+|n|)^{-(j+1)}\left|\alpha_{n}^{11}\right| \sum_{m=1}^{\infty}\left|A_{n m}^{11}\right|\left(m+n+\frac{1}{2}\right)^{t}+\left|A_{n m}^{12}\right|(m+n)^{t}\right)^{2} \\
& \leq G \sum_{n=1}^{\infty} \exp \left(-\varepsilon \frac{\sqrt{2}}{4} n^{1 / 2}\right)(1+|n|)^{-2(j+1)}<\infty .
\end{aligned}
$$

Secondly, using (3.30) and (3.31) we obtain that

$$
\begin{aligned}
\sum_{n=1}^{\infty} 2\{ & {\left[\sum_{t=0}^{j}\left|\alpha_{n}^{11}\right|\left|C_{t}\right|(1+|n|)^{-(j+1)}\left(n+\frac{1}{2}\right)^{t}\right] } \\
& \left.\times\left[\sum_{t=0}^{j}\left|C_{t}\right|\left|\alpha_{n}^{11}\right| \sum_{m=1}^{\infty}(1+|n|)^{-(j+1)}\left(\left(m+n+\frac{1}{2}\right)^{t}\left|A_{n m}^{11}\right|+(m+n)^{t}\left|A_{n m}^{12}\right|\right)\right]\right\} \\
\leq & \sum_{n=1}^{\infty}\left[\sum_{t=0}^{j}(1+|n|)^{-2(j+1)}\left(n+\frac{1}{2}\right)^{t} \exp \left(-\varepsilon \frac{\sqrt{2}}{4} n^{1 / 2}\right) G^{1 / 2}\right]<\infty
\end{aligned}
$$

and also the first part of the (3.31) obviously convergent so, we get from (3.33) and (3.34)

$$
\sum_{n \in \mathbb{N}}(1+|n|)^{-2(j+1)} \frac{1}{(j !)^{2}}\left(\sum_{t=0}^{j}\left|C_{t}\left\{\frac{d^{t}}{d \lambda^{t}} f_{n}^{(1)}\left(z_{i}\right)\right\}\right|\right)^{2}<\infty
$$


and similarly

$$
\sum_{n \in \mathbb{N}}(1+|n|)^{-2(j+1)} \frac{1}{(j !)^{2}}\left(\sum_{t=0}^{j}\left|D_{t}\left\{\frac{d^{t}}{d \lambda^{t}} f_{n}^{(2)}\left(z_{i}\right)\right\}\right|\right)^{2}<\infty
$$

Finally $d^{j} /\left(d \lambda^{j}\right) V_{n}\left(\lambda_{i}\right) \in H_{-(j+1)}(\mathbb{N}), j=0,1,2, \ldots, m_{i}-1, i=k+1, k+2, \ldots, v$.

\section{References}

[1] M. A. NaImark, "Investigation of the spectrum and the expansion in eigenfunctions of a nonselfadjoint differential operator of the second order on a semi-axis," American Mathematical Society Translations, vol. 16, pp. 103-193, 1960.

[2] V. E. Lyance, "A differential operator with spectral singularities, I, II," American Mathematical Society Translations, vol. 2, no. 60, pp. 185-225, 1967.

[3] M. Adıvar, "Quadratic pencil of difference equations: jost solutions, spectrum, and principal vectors," Quaestiones Mathematicae, vol. 33, no. 3, pp. 305-323, 2010.

[4] M. Adivar and M. Bohner, "Spectral analysis of $q$-difference equations with spectral singularities," Mathematical and Computer Modelling, vol. 43, no. 7-8, pp. 695-703, 2006.

[5] M. Adivar and M. Bohner, "Spectrum and principal vectors of second order $q$-difference equations," Indian Journal of Mathematics, vol. 48, no. 1, pp. 17-33, 2006.

[6] E. Bairamov and A. O. Çelebi, "Spectrum and spectral expansion for the non-selfadjoint discrete Dirac operators," The Quarterly Journal of Mathematics, vol. 50, no. 200, pp. 371-384, 1999.

[7] E. Bairamov and C. Coskun, "Jost solutions and the spectrum of the system of difference equations," Applied Mathematics Letters, vol. 17, no. 9, pp. 1039-1045, 2004.

[8] E. Bairamov, Y. Aygar, and T. Koprubasi, "The spectrum of eigenparameter-dependent discrete SturmLiouville equations," Journal of Computational and Applied Mathematics, vol. 235, no. 16, pp. 4519-4523, 2011.

[9] M. Adivar and E. Bairamov, "Spectral properties of non-selfadjoint difference operators," Journal of Mathematical Analysis and Applications, vol. 261, no. 2, pp. 461-478, 2001.

[10] E. Bairamov and T. Koprubasi, "Eigenparameter dependent discrete Dirac equations with spectral singularities," Applied Mathematics and Computation, vol. 215, no. 12, pp. 4216-4220, 2010. 




Advances in

Operations Research

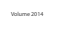



\section{The Scientific} World Journal
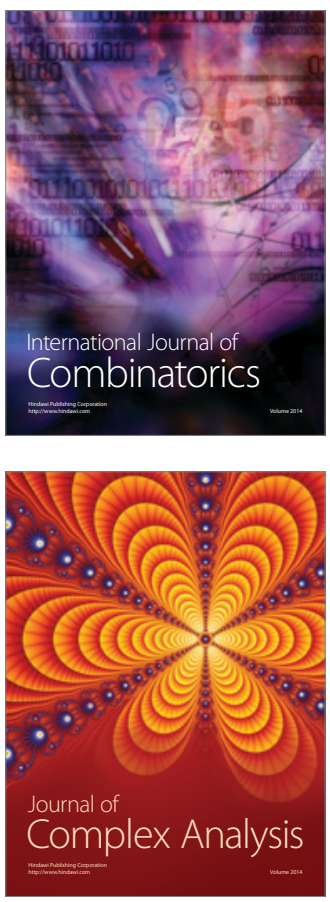

International Journal of

Mathematics and

Mathematical

Sciences
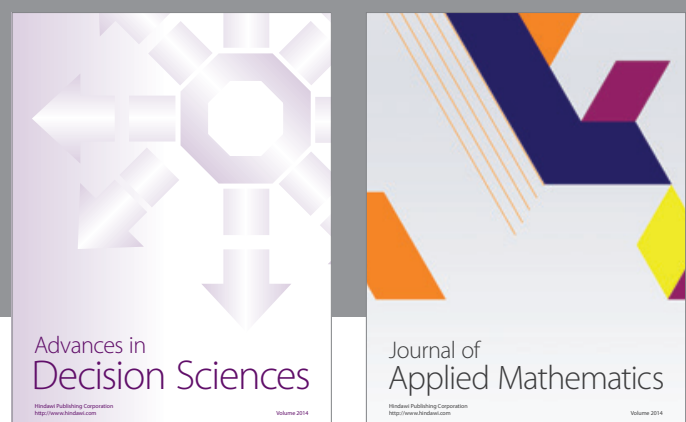

Journal of

Applied Mathematics


Submit your manuscripts at http://www.hindawi.com


Mathematical Problems in Engineering
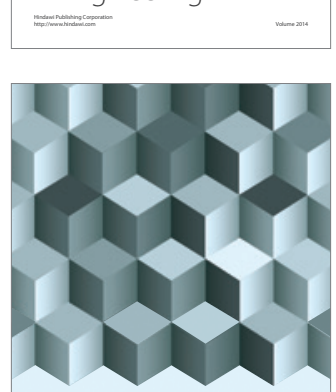

Journal of

Function Spaces
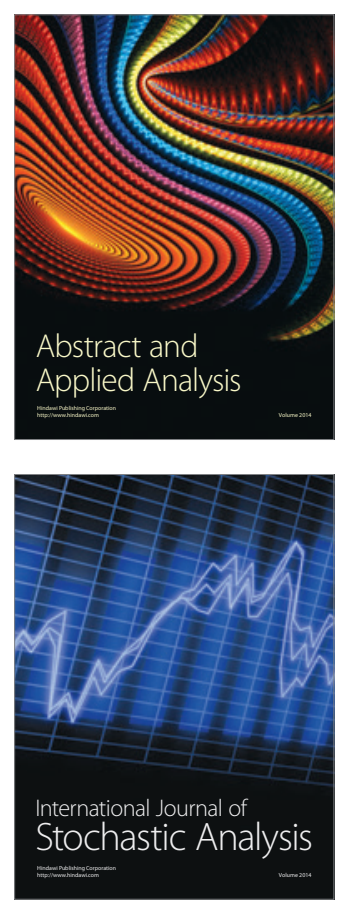



ournal of

Probability and Statistics

Promensencen
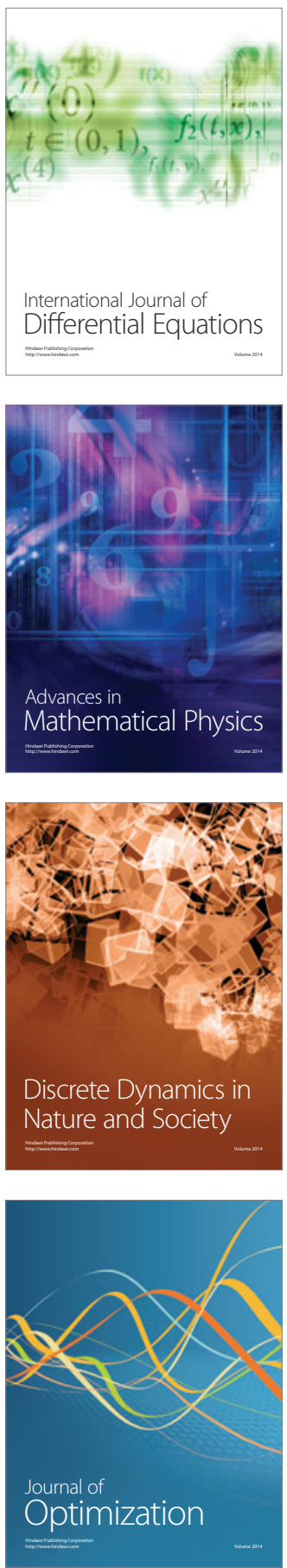